\title{
Safety and Effectiveness of Sacroplasty: A Large Single-Center Experience
}

A.C. Gupta, R.V. Chandra, A.J. Yoo, T.M. Leslie-Mazwi, D.L. Bell, B.P. Mehta, T.L. Vanderboom, J.D. Rabinov, M. Larvie, and J.A. Hirsch

\begin{abstract}
BACKGROUND AND PURPOSE: Sacral insufficiency fractures are a common cause of severe low back pain and immobilization in patients with osteoporosis or cancer. Current practice guideline recommendations range from analgesia and physical therapy to resection with surgical fixation. We sought to assess the safety and effectiveness of sacroplasty, an emerging minimally invasive treatment.
\end{abstract}

MATERIALS AND METHODS: We performed a retrospective review of institutional databases for percutaneous sacroplasty performed between January 2004 and September 2013. Demographic and procedural data and pre- and posttreatment Visual Analog Scale, Functional Mobility Scale, and Analgesic Scale scores were reviewed. Overall response was rated by using a 4-point scale (1, complete resolution of pain; 2, improvement of pain; 3, no change; 4 , worsened pain) assessed at short-term follow-up.

RESULTS: Fifty-three patients were included; most (83\%) were female. Fracture etiology was cancer-related (55\%), osteoporotic insufficiency (30\%), and minor trauma (15\%). No major complication or procedure-related morbidity occurred. There were statistically significant decreases in the Visual Analog Scale $(P<.001)$, Functional Mobility Scale $(P<.001)$, and Analgesic Scale scores $(P<.01)$ in 27 patients with recorded data: pretreatment Visual Analog Scale (median [interquartile range], 9.0 [8.0-10.0]); Functional Mobility Scale, 3.0 (2.0-3.0); and Analgesic Scale scores, 3.0 (3.0-4.0) were reduced to $3.0(0.0-5.8)$, 1.0 (0.25-2.8), and $3.0(2.0-3.8)$ posttreatment. When we used the overall 4-point score at a mean of 27 days, $93 \%(n=45)$ reported complete resolution or improvement in overall pain.

CONCLUSIONS: In this single-center cohort, sacroplasty was a safe and effective procedure. There were significant short-term gains in pain relief, increased mobility, and decreased dependence on pain medication.

ABBREVIATIONS: AS = Analgesic Scale; FMS = Functional Mobility Scale; $\mathrm{PMMA}=$ polymethylmethacrylate; $\mathrm{VAS}=$ Visual Analog Scale

S acral insufficiency fractures are a common source of debilitating pain and immobilization in the elderly population. Typically seen in the sacral ala or S2 vertebral body, they may result from osteopenia, osteolysis from cancer metastasis (specifically postradiation therapy), or trauma. ${ }^{1}$ Most commonly seen in the osteoporotic elderly female population, sacral insufficiency fractures tend to present with nonlocalized low back pain with or without radiation to the buttocks. They can be difficult to diagnose because most nondisplaced or transverse sacral fractures are not clearly identified on conventional radiology. Specifically, they require more advanced imaging with $\mathrm{CT}, \mathrm{MR}$ imaging, or nuclear

Received February 5, 2014; accepted after revision April 16.

From the Department of Radiology (A.C.G., A.J.Y., T.M.L.-M., D.L.B., B.P.M., T.L.V., J.D.R., M.L., J.A.H.), Massachusetts General Hospital, Harvard Medical School, Boston, Massachusetts; and Department of Radiology (R.V.C.), Monash Health, and Department of Surgery, Monash University, Melbourne, Australia.

Please address correspondence to Joshua A. Hirsch, MD, Department of Radiology, Massachusetts General Hospital, Harvard Medical School, Gray 241, 55 Fruit St, Boston, MA 02114; e-mail: jahirsch@partners.org, jahirsch@mgh.harvard.edu

http://dx.doi.org/10.3174/ajnr.A4027 bone scintigraphy for accurate diagnosis. ${ }^{2}$ As a result, detection may be delayed for many weeks. ${ }^{1}$

The current standard of care is a short period of bed rest and analgesia, often with opioid analgesics. Physical therapy to facilitate an early return to mobilization is encouraged to avoid physical deconditioning and associated complications of immobilization (venous thrombosis, pulmonary embolism, and pressure sores). Surgical fixation is generally reserved for patients with displaced dorsal fractures or fracture dislocations. For some patients, a prolonged conservative approach carries significant risk of morbidity from immobilization and opioid side effects such as respiratory depression, change in mental status, and dependence. ${ }^{3}$

Percutaneous sacroplasty has emerged more recently as a therapeutic option to reduce pain and facilitate early mobilization. While there are no randomized controlled trial data to support its efficacy, observational studies have reported significant reductions in pain scores and narcotic analgesic requirements, with an acceptable safety profile. ${ }^{4-11}$ Data on functional mobility outcomes are more limited. The aim of this study was to evaluate the 

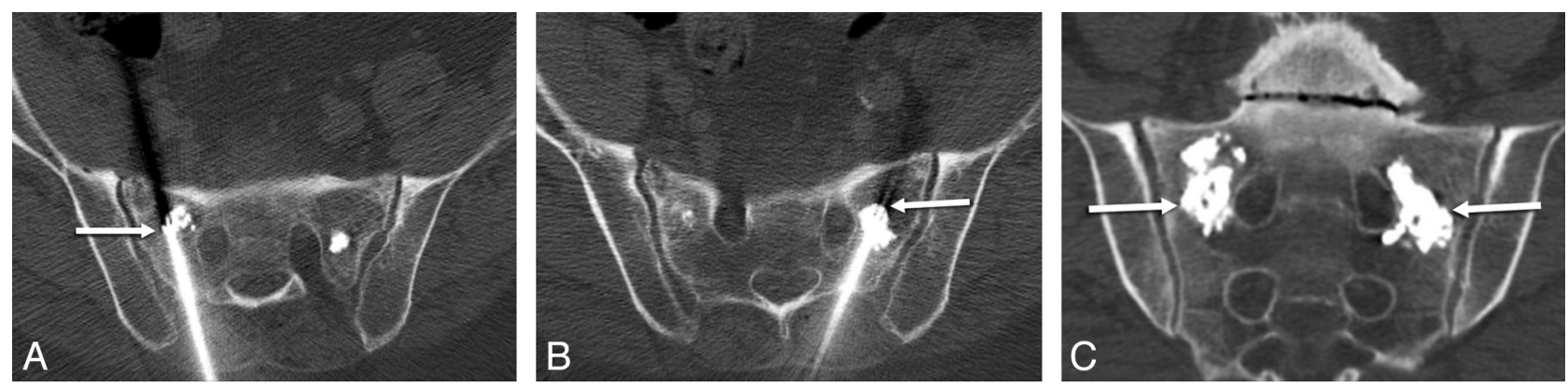

FIG 1. Percutaneous CT-guided sacroplasty. Intraprocedural axial CT images during PMMA injection ( $A$ and $B)$. The needle tip position is identified by the presence of beam-hardening artifacts (arrows) with adjacent PMMA deposition. Final postprocedural coronal CT image (C) reveals satisfactory PMMA deposition (arrows), with no extravasation into the sacral neural foramina.

Table 1: Functional Mobility Scale and Analgesic Scale ${ }^{13}$

\begin{tabular}{cll}
\hline Score & \multicolumn{1}{c}{ Functional Mobility Scale } & \multicolumn{1}{c}{ Analgesic Scale } \\
\hline 0 & Full activity & No pain medication use \\
1 & Walking with assistance & Aspirin, acetaminophen (Tylenol), nonsteroidal anti-inflammatory drugs \\
2 & Walking with assistance for short periods & Prescription nonnarcotics \\
3 & Walking with assistance for activities of daily living/appointments only & Oral narcotic as needed \\
4 & Confined to a wheelchair & Oral narcotic scheduled \\
5 & Bedridden & Parental narcotic \\
\hline
\end{tabular}

Reproduced from Gupta AC, Hirsch JA, Chaudhry ZA, et al. Evaluating the safety and effectiveness of percutaneous acetabuloplasty. J Neurointerv Surg 2012;4:134-38, with permission from BMJ Publishing Group Ltd.

safety and effectiveness of sacroplasty in a large single-center cohort, with emphasis on overall response to pain, functional mobility, and analgesic use.

\section{MATERIALS AND METHODS \\ Patient Cohort}

Under institutional review board approval, we retrospectively reviewed institutional databases to identify percutaneous sacroplasty cases performed at our institution from January 2004 to September 2013. Patients were referred for pain relief after a poor response to conservative therapy. Chart review was performed to obtain patient demographic data and fracture etiology. In patients with non-cancer-related fractures, fracture etiology was defined as osteoporotic insufficiency unless there was a clear history of a preceding traumatic event, such as a mechanical fall. In these cases, fracture etiology was deemed traumatic. All patients were evaluated with either CT or MR imaging before the procedure to assess fracture characteristics, extent of bony involvement, and proximity to neural structures. For cancer-related fractures, only patients with osteolytic or mixed osteolytic/osteoblastic lesions were included. Patients with osteoblastic primary tumors or predominantly osteoblastic metastasis were excluded due to the anticipated difficulty of needle placement. In addition, patients with involvement of the sacroiliac joint, clear osseous breach of the sacral neural foramina, or local neural compression were also excluded. Informed consent was obtained before the procedure, and Health Insurance Portability and Accountability Act compliance was maintained.

\section{Technique}

Procedures were performed with intravenous sedation, typically by using a combination of fentanyl and midazolam or with the patient under general anesthesia. Local anesthetic was infiltrated in the skin, subcutaneous tissues, and periosteum. Continuous $\mathrm{x}$-ray fluoroscopy, CT fluoroscopy, or intermittent CT scanning was used to place an 11-gauge AVAmax needle (CareFusion, San Diego, California) into the fracture site by using either a shortaxis or posterior long-axis approach. No adjunctive cavity creation (curettage and/or balloon inflation) or tumor ablative therapies (radiofrequency ablation/laser/cryotherapy) were used. Polymethylmethacrylate (PMMA) cement (Advanced Biomaterial Systems, Chatham, New Jersey) was then mixed and injected through the trocar by using a screw injector under continuous $\mathrm{x}$-ray fluoroscopic guidance or intermittent CT scanning to monitor cement deposition. For CT-guided procedures, a small volume of PMMA was injected, and then a limited CT that covered the target site was performed to ensure that there was no untoward craniocaudal cement migration before injecting further PMMA (Fig 1). Using this intermittent CT method, we aimed to prevent extravasation into the sacral neural foramina. Final postprocedural images were used to evaluate final filling of the lesion and assess technical complications.

\section{Outcomes}

Charts were reviewed to identify procedural complication data. Significant complications were defined as cement leakage leading to permanent neurologic deficit, cauda equina syndrome, a new neurologic deficit requiring surgical intervention, or systemic complications such as pulmonary embolism or procedure-related mortality.

The Visual Analog Scale (VAS), Functional Mobility Scale (FMS), and Analgesic Scale (AS) were used for pre- and postprocedural assessments. The VAS is a widely validated pain assessment tool that correlates sketched faces with numbers from 0 to 10 , with 0 representing no pain to 10 representing the worst pain the patient has ever experienced. ${ }^{12}$ The FMS measures mobility of the patient, with ranges from 0 to 5 , with 0 representing full activity and 5 representing a bedridden patient (Table 1). The AS assesses a patient's use of pain medication on a scale from 0 to 5 , with 0 representing no medication use and 5 representing paren-

AJNR Am J Neuroradiol 35:2202-06 Nov 2014 www.ajnr.org 
Table 2: Four-level pain scale ${ }^{9}$

\begin{tabular}{cl}
\hline Score & \multicolumn{1}{c}{ Four-Level Pain Scale } \\
\hline 1 & Complete pain resolution \\
2 & Improvement in fracture-related pain \\
3 & No change in pain \\
4 & Worsening of pain after the procedure \\
\hline
\end{tabular}

Table 3: Summary data for 53 patients who underwent percutaneous sacroplasty

\begin{tabular}{lc}
\multicolumn{1}{c}{ Characteristics } & Data \\
\hline Total patients & 53 \\
Age (mean) (yr) & $76.4 \pm 2.8$ \\
Age younger than 70 yr & $13(25 \%)$ \\
Age 70-79 yr & $20(38 \%)$ \\
Age 80-89 yr & $15(28 \%)$ \\
Age 90 yr or older & $5(9 \%)$ \\
Female & $44(83 \%)$ \\
Female/male ratio & $5: 1$ \\
Cancer-related fracture & $29(55 \%)$ \\
Breast cancer & $9 / 29(31 \%)$ \\
Multiple myeloma & $7 / 29(24 \%)$ \\
Colorectal cancer & $6 / 29(21 \%)$ \\
Small cell lung cancer & $2 / 29(7 \%)$ \\
Prostate cancer & $1 / 29(3 \%)$ \\
Melanoma & $1 / 29(3 \%)$ \\
Cervical cancer & $1 / 29(3 \%)$ \\
Hematologic malignancy & $1 / 29(3 \%)$ \\
Pancreatic cancer & $1 / 29(3 \%)$ \\
Osteoporotic fracture & $16(30 \%)$ \\
Traumatic fracture & $8(15 \%)$ \\
Bilateral fractures treated & $44(83 \%)$ \\
Sacroplasty with conscious sedation & $39(74 \%)$ \\
Sacroplasty under general anesthesia & $14(26 \%)$ \\
Sacroplasty with CT guidance & $48(91 \%)$ \\
Sacroplasty with fluoroscopic guidance & $5(9 \%)$ \\
Significant complications & 0 \\
Procedural mortality & 0 \\
Time to follow-up (days) & $27( \pm 3.7)$ \\
\hline
\end{tabular}

tal narcotic use (Table 1). Both the FMS and the AS have been described in the evaluation of cement augmentation procedures. ${ }^{13}$ Formal, documented VAS, FMS, and AS evaluations became routine during the study period and were performed between January 2007 and September 2013. A 4-level pain scale was also used to evaluate patient response to the procedure (Table 2). ${ }^{9}$ The scale ranges from 1 to 4 and was used to measure patient response to the procedure following sacroplasty, where 1 represents complete resolution of pain; 2, improvement of pain; 3 , no change; and 4, worsened pain following the procedure.

\section{Statistical Analysis}

All summary statistics for continuous variables were reported as mean ( \pm SD and range), while categoric variables were reported as percentages. VAS, FMS, and AS scores (ordinal variables) were reported as median (interquartile range). Normality was assessed by using the Kolmogorov-Smirnov test. The Wilcoxon signed rank test was performed for statistical correlation with paired testing of preand posttreatment scores. Statistical significance was defined as $P<$ .05. Statistical analysis was performed with MedCalc for Windows software, Version 12.7.7 (MedCalc Software, Mariakerke, Belgium).

\section{RESULTS}

Fifty-three patients met the study criteria. Table 3 outlines the
Table 4: Pre- and posttreatment VAS, FMS, and AS scores reported as median (interquartile range)

\begin{tabular}{lccc}
\hline & Preprocedure & Postprocedure & $\boldsymbol{P}$ Value \\
\hline Visual Analog Scale & $9.0(8.0-10.0)$ & $3.0(0.0-5.8)$ & $<.001$ \\
Functional Mobility Scale & $3.0(2.0-3.0)$ & $1.0(0.25-2.8)$ & $<.001$ \\
Analgesic Scale & $3.0(3.0-4.0)$ & $3.0(2.0-3.8)$ & $<.01$ \\
\hline
\end{tabular}

Table 5: Pre- and posttreatment VAS, FMS, and AS scores for noncancer fractures (osteoporotic insufficiency and traumatic cohort combined) reported as median (interquartile range)

\begin{tabular}{lccc}
\hline & Preprocedure & Postprocedure & $\boldsymbol{P}$ Value \\
\hline Visual Analog Scale & $9.5(8.0-10.0)$ & $0.0(0.0-4.0)$ & $<.001$ \\
Functional Mobility Scale & $3.0(1.5-3.0)$ & $1.0(0.0-2.0)$ & $<.01$ \\
Analgesic Scale & $3.0(3.0-3.0)$ & $3.0(1.0-3.0)$ & .06 \\
\hline
\end{tabular}

Table 6: Pre- and posttreatment VAS, FMS, and AS scores for cancer-related fractures reported as median (interquartile range)

\begin{tabular}{lccc}
\hline & Preprocedure & Postprocedure & $\boldsymbol{P}$ Value \\
\hline Visual Analog Scale & $8.0(8.0-10.0)$ & $4.0(2.3-6.0)$ & $<.01$ \\
Functional Mobility Scale & $3.0(2.3-3.8)$ & $2.0(1.0-3.0)$ & .11 \\
Analgesic Scale & $4.0(3.0-4.0)$ & $4.0(3.0-4.0)$ & .19 \\
\hline
\end{tabular}

summary data for the total cohort. Mean age was 76 years; most were female $(83 \%)$ patients with either cancer-related $(55 \%)$ or osteoporotic insufficiency (30\%) fractures. Breast carcinoma metastasis, multiple myeloma, and colorectal carcinoma metastasis accounted for three-quarters of cancer-related fractures. All traumatic fractures were the result of minor trauma, such as a mechanical fall. No patients with high-energy trauma such as a motor vehicle collision were included. Fifteen of the 53 patients had vertebral augmentation before sacroplasty; however, none had these procedures performed within 3 months of sacroplasty, and most $(80 \%)$ were performed $>9$ months before sacroplasty.

Percutaneous sacroplasty was generally performed with the patient under conscious sedation (74\%) and by using CT guidance $(91 \%)$. Bilateral fractures were treated in 44 patients. For the total cohort of 53 patients with 97 sacral fractures treated, no significant complications occurred. There was no procedural mortality. Effectiveness was assessed by using the VAS, FMS, AS,

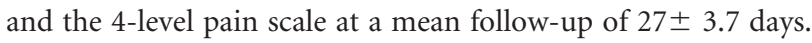
Pre- and posttreatment VAS, FMS, and AS scores were available for 27 patients. There were statistically significant reductions in VAS, FMS, and AS scores after sacroplasty (Table 4). Pretreatment median (interquartile range) VAS, 9.0 (8.0-10); FMS, 3.0 (2.0-3.0); and AS, 3.0 (3.0-4.0) were reduced to median posttreatment (interquartile range) VAS, $3.0(0.0-5.8)$; FMS, 1.0 (0.25-2.8); and AS 3.0 (2.0-3.8). Nineteen of the 53 patients had additional pelvic fractures at the time of presentation, mostly pubic rami fractures. In this smaller cohort of patients with additional pelvic fractures, all had significant reduction in their VAS, FMS, and AS scores after sacroplasty.

Osteoporotic insufficiency fractures were combined with traumatic fractures to form the noncancer fracture cohort to facilitate comparison of outcomes according to fracture etiology. Before treatment, there was no significant difference in the VAS $(P=.94)$, FMS $(P=.74)$, or AS $(P=.11)$ scores between the noncancer- $(n=16)$ and cancer-related fracture cohorts $(n=$ 11). The treatment effect of sacroplasty in the noncancer and cancer-related fracture cohorts are summarized in Tables 5 and 6. 


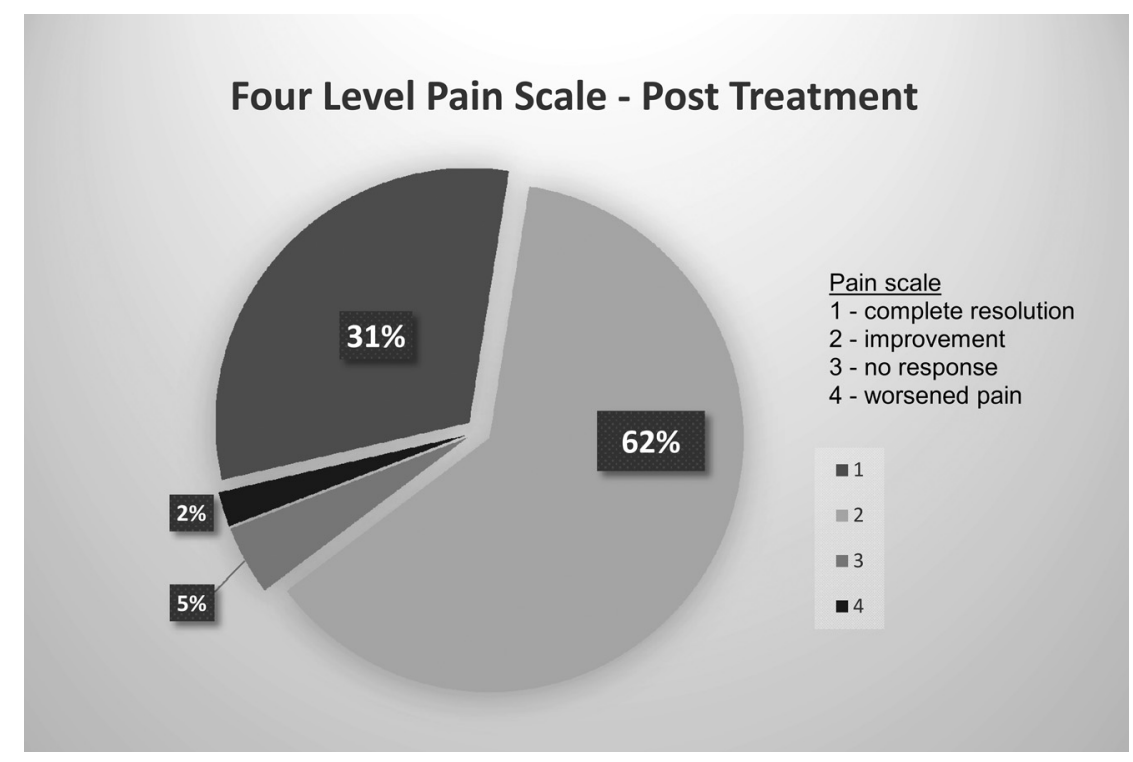

FIG 2. Treatment effect of sacroplasty on the 4-level pain scale scores at 1 month. Most patients (93\%) reported improvement or complete resolution of pain.
Our study confirms this treatment effect of percutaneous sacroplasty on pain and analgesic use. Postulated mechanisms of pain relief include increased structural stability in a previously weakened weight-bearing site after cement deposition and neurolysis of painful nerve fibers. ${ }^{16,17}$

There are little data on functional mobility outcomes after percutaneous sacroplasty. A single study assessed 1-month ambulation outcomes after sacroplasty by using a 3-point scale: 1 , worse; 2, unchanged; and 3, improvement of ambulation. Most impressively, $80 \%$ with walking limitation experienced improvement. ${ }^{7}$ We add to the emerging literature on sacroplasty by reporting quantifiable and significant short-term improvement in functional mobility by using the FMS.

In our cohort, patients with osteopo-

The reduction in median VAS and AS scores was significantly greater for the non-cancer-related fractures $(P=.02$ and $P=.004$ respectively) compared with the cancer-related fractures. There were no significant differences in the reduction of median FMS in non-cancer-related and cancer-related fracture cohorts.

Four-level pain scale scores were available for 45 patients. After sacroplasty, $93 \%$ reported complete resolution or improvement in overall pain. Almost a third $(n=14)$ reported complete resolution of pain. Only 2 patients had no improvement, and 1 patient had worsened pain after sacroplasty (Fig 2). Two patients underwent vertebral augmentation after sacroplasty. Both of these patients reported either improvement or complete resolution of their pain on the 4-level pain scale at short-term follow-up before new symptomatic thoracic vertebral compression fractures occurred.

\section{DISCUSSION}

Sacral insufficiency fractures are severely debilitating to the elderly population, exacerbated by delays to diagnosis and the limited treatment options available. Percutaneous sacroplasty is a minimally invasive therapeutic strategy for patients not responding to conservative therapy with bed rest, physical therapy, and analgesics or for those who are not surgical candidates. Our results show that percutaneous sacroplasty effectively decreases pain, increases mobility, and decreases analgesic requirements in patients with sacral fractures. The quantified improvements in pain, mobility, and anesthesia are supported by the dramatic response in the 4-point scale score-almost one-third had complete resolution of their pain; 93\% reported improvement or complete resolution.

To date, there have been several case series showing good outcomes following sacroplasty. ${ }^{4-8,14}$ The largest multicenter cohort to date included 243 patients and showed that most patients responded positively to sacroplasty and had statistically significant decreases in pain based on the VAS. ${ }^{6}$ A recent single-center cohort of 57 patients also showed reduced opioid use after sacroplasty. ${ }^{15}$ rotic insufficiency or minor traumatic fractures gained greater reductions in VAS and AS scores compared with patients with cancer-related fractures. These results are similar to those from the largest multicenter cohort. ${ }^{6}$ This finding may be, in part, due to the presence of additional fractures that may have impacted outcome. However, in the small cohort of patients with additional pelvic fractures (mainly pubic rami), all had a significant reduction in their VAS, FMS, and AS scores, suggesting that the presence of additional fractures did not impact the short-term effect of sacroplasty.

The treatment effectiveness for an individual patient is balanced by the procedural safety. In the large multicenter cohort of 243 patients, there were no significant cement extravasations, infections, hemorrhages, pulmonary emboli, or procedure-related deaths. ${ }^{6}$ This outcome may be, in part, due to the use of CT guidance and the limitation to operators trained and experienced in vertebral augmentation techniques. Similarly, we performed most procedures (91\%) under CT guidance, and we limited performance of sacroplasty to operators trained and experienced in vertebral augmentation and acetabuloplasty. No complications occurred in our cohort. We thus confirm that when performed by experienced operators, percutaneous sacroplasty is a safe procedure.

Our study has significant limitations, in particular the lack of uniform patient response in the VAS, FMS, and AS scores. Documentation of these scores became routine during the study period. However, we sought to rectify the lower rate of recorded outcome scores for VAS, FMS, and AS by including the 4-level pain scale score. The inclusion of pain improvement and pain worsening allows more useful interpretation of the patient perspective on treatment effectiveness compared with binary yes or no responses. While we have demonstrated significant short-term gains, other studies have shown durable pain reduction for up to 1.5 years. ${ }^{11,18,19}$

Ultimately, percutaneous sacroplasty should be evaluated in a 
randomized controlled trial against conservative therapy with analgesia and/or surgical fixation. Until then, emerging data from our study can be included in comparative effectiveness research to define treatment appropriateness criteria for percutaneous sacroplasty and to inform health care decisions on the relative benefits and harm of the different treatment options available for patients with sacral fractures.

\section{CONCLUSIONS}

Sacral insufficiency fractures continue to be a frequent source of pain and disability in the elderly population. Percutaneous sacroplasty effectively decreases pain, increases mobility, and decreases analgesic requirements in patients with painful sacral fractures. Complications are rare with appropriate techniques and operator experience. Percutaneous sacroplasty is an excellent treatment option for patients with painful sacral fractures not responding to conservative therapy.

Disclosures: Joshua A. Hirsch-RELATED: Consulting Fees or Honoraria: from CareFusion, a company that makes augmentation products that could be used in sacroplasty; Stocks/Stock Options: He also holds stocks/stock options in Intratech.

\section{REFERENCES}

1. Gotis-Graham I, McGuigan L, Diamond T, et al. Sacral insufficiency fractures in the elderly. J Bone Joint Surg Br 1994;76:882-86

2. Coleman RE. Clinical features of metastatic bone disease and risk of skeletal morbidity. Clin Cancer Res 2006;12:6243s-49s

3. Babayev M, Lachmann E, Nagler W. The controversy surrounding sacral insufficiency fractures: to ambulate or not to ambulate? Am J Phys Med Rehabil 2000;79:404-09

4. Gupta AC, Yoo AJ, Stone J, et al. Percutaneous sacroplasty. J Neurointerv Surg 2012;4:385-89

5. Hirsch JA, Barr JD, Zoarski GH. Sacroplasty: beyond the beginning. J Neurointerv Surg 2013;5:395

6. Kortman K, Ortiz O, Miller T, et al. Multicenter study to assess the efficacy and safety of sacroplasty in patients with osteoporotic sacral insufficiency fractures or pathologic sacral lesions. J Neurointerv Surg 2013;5:461-66
7. Pereira LP, Clarencon F, Cormier E, et al. Safety and effectiveness of percutaneous sacroplasty: a single-centre experience in 58 consecutive patients with tumours or osteoporotic insufficient fractures treated under fluoroscopic guidance. Eur Radiol 2013;23:2764-72

8. Cho CH, Mathis JM, Ortiz O. Sacral fractures and sacroplasty. Neuroimaging Clin N Am 2010;20:179-86

9. Jha RM, Yoo AJ, Hirsch AE, et al. Predictors of successful palliation of compression fractures with vertebral augmentation: single-center experience of 525 cases. J Vasc Interv Radiol 2009;20:760-68

10. Whitlow CT, Yazdani SK, Reedy ML, et al. Investigating sacroplasty: technical considerations and finite element analysis of polymethylmethacrylate infusion into cadaveric sacrum. AJNR Am J Neuroradiol 2007;28:1036-41

11. Frey ME, Depalma MJ, Cifu DX, et al. Percutaneous sacroplasty for osteoporotic sacral insufficiency fractures: a prospective, multicenter, observational pilot study. Spine J 2008;8:367-73

12. DeLoach LJ, Higgins MS, Caplan AB, et al. The visual analog scale in the immediate postoperative period: intrasubject variability and correlation with a numeric scale. Anesth Analg 1998;86:102-06

13. Gupta AC, Hirsch JA, Chaudhry ZA, et al. Evaluating the safety and effectiveness of percutaneous acetabuloplasty. J Neurointerv Surg 2012;4:134-38

14. Trouvin AP, Alcaix D, Somon T, et al. Analgesic effect of sacroplasty in osteoporotic sacral fractures: a study of six cases. Joint Bone Spine 2012;79:500-03

15. Dougherty RW, McDonald JS, Cho YW, et al. Percutaneous sacroplasty using CT guidance for pain palliation in sacral insufficiency fractures. J Neurointerv Surg 2014;6:57-60

16. Harty JA, Brennan D, Eustace S, et al. Percutaneous cementoplasty of acetabular bony metastasis. Surgeon 2003;1:48-50

17. Richards AM, Mears SC, Knight TA, et al. Biomechanical analysis of sacroplasty: does volume or location of cement matter? AJNR Am J Neuroradiol 2009;30:315-17

18. Whitlow CT, Mussat-Whitlow BJ, Mattern CW, et al. Sacroplasty versus vertebroplasty: comparable clinical outcomes for the treatment of fracture-related pain. AJNR Am J Neuroradiol 2007;28: 1266-70

19. Kamel EM, Binaghi S, Guntern D, et al. Outcome of long-axis percutaneous sacroplasty for the treatment of sacral insufficiency fractures. Eur Radiol 2009;19:3002-07 